\title{
Botany
}

\section{Embryo position of Castanopsis sclerophylla (Fagaceae) seeds with recalcitrant storage behavior differs from Quercus genus, but response to desiccation shows no difference}

\begin{tabular}{|r|l|}
\hline Journal: & Botany \\
\hline Manuscript ID & cjb-2021-0081.R2 \\
\hline Manuscript Type: & Note \\
\hline Date Submitted by the & 30 -Aug-2021 \\
\hline $\begin{array}{r}\text { Complete List of Authors: } \\
\text { Keyword: }\end{array}$ & $\begin{array}{l}\text { Li, Jiajin; University of Shanghai for Science and Technology, Institute of } \\
\text { Jaganathan, Ganesh; University of Shanghai for Science and Technology } \\
\text { Kang, Han; University of Shanghai for Science and Technology } \\
\text { Liu, Baolin; University of Shanghai for Science and Technology, Institute } \\
\text { of Biothermal Engineering }\end{array}$ \\
\hline $\begin{array}{r}\text { Is the invited manuscript for } \\
\text { consideration in a Special } \\
\text { Issue? : }\end{array}$ & \begin{tabular}{l} 
Not applicable (regular submission) \\
\hline
\end{tabular} \\
\hline
\end{tabular}

\section{SCHOLARONE ${ }^{m}$ \\ Manuscripts}



Shanghai 200093, China.

ॠEqual first authors

8

Email: jganeshcbe@gmail.com 


\section{Abstract} In many -but not all- recalcitrant Quercus seeds, water loss during drying only occurs through the scar. Consequently, the embryo axis sitting on the opposite side of the scar is well protected from desiccation. However, whether such a mechanism is common throughout Fagaceae species is unexplored. Similarly,

27 little is known about the desiccation response of other Fagaceae genera. Germination and desiccation tolerance of Castanopsis sclerophylla (Fagaceae) were studied by drying the seeds with silica gel. Fresh seeds had a moisture content (MC) of $36 \%$ and germinated to $92.5 \%$ when incubated at $15 / 20^{\circ} \mathrm{C}$. Drying seeds to 22.5 and $20.7 \%$ MC decreased germination to 27 and 5\%, respectively; indicating that seeds are recalcitrant. X-ray computed tomography and photomicrographs showed that the embryo axis of $C$. sclerophylla is located just below the scar. Regardless of drying occurring either through only scar or the whole pericarp, embryo drying was deemed unavoidable. Nevertheless, germination percentage during drying showed exceptional similarities with the other Fagaceae species. 
Based on their ability to withstand drying, two broad groups of seeds have been recognised by Roberts (1973). One group includes nearly $90 \%$ of the known plant species with seeds that can survive drying to the low moisture content (MC), c. 3-10\% on a fresh weight basis (f. wb), thus they are desiccationtolerant, i.e. orthodox (Wyse and Dickie, 2016). Another group of species produces seeds that lose viability if dried to the $\mathrm{MC}$ range of $20-30 \%$ f.wb, therefore they are referred to as desiccation-sensitive or recalcitrant (Roberts, 1973). The seeds of desiccation-sensitive species are not amendable to low temperature storage e.g. $-20^{\circ} \mathrm{C}$ in seed bank and storing them at higher temperatures in the range of 0 $10^{\circ} \mathrm{C}$ does not preserve viability for more than 1 year (Berjak and Pammenter, 2008).

The family Fagaceae is widely distributed in the temperate forests and includes nine genera with approximately c. 1000 species. Information on seed storage behavior and germination ecology of Fagaceae seeds mostly is restricted to the largest genus Quercus, which includes c. 400 species (Bonner, 1996; Xia et al., 2012a). These studies suggest that most Quercus seeds are large, they do not withstand drying to MC less than $15-20 \%$ f. wb and are recalcitrant. However, the presence of desiccation tolerance has been observed in other genera within the Fagaceae, e.g. Fagus (Suszka and Zieta, 1977; SID, 2020). Surprisingly, little is known about the seed storage behavior of species in other genera within Fagaceae, making it an important family to study the response of seeds of desiccation. Castanopsis is the second largest genus in Fagaceae, mainly distributed in the subtropical and temperate region of Asia, and it often grows with other genera of Fagaceae such as Quercus to form a pure "oak forest" (Huang et al., 1999). Castanopsis includes approximately 120 species, and there are 63 species in China, of which 30 are endemic (Huang et al., 1999; Wu et al., 2014). Current knowledge about seed storage behavior of Castanopsis species is unclear. For example, Kew's seed information database (SID, 
67

2020) reports seed storage behavior of 10 Castanopsis species, yet 6 of them are labelled as uncertain and 4 species (1 orthodox and 3 recalcitrant) are doubtfully assigned.

Such confusion is further complicated by previous studies lacking desiccation experiments or failing to report the MC. Schopmeyer (1974), for example, reported that only 5\% of the seeds of Castanopsis chryophylla Dougl. were viable following hermetic air-dry storage at $5^{\circ} \mathrm{C}$ for 5 years, but the $\mathrm{MC}$ of the seeds was not mentioned. Similarly, Campbell (1980) showed that seeds of $C$. indica Roxb, $C$. purpurella Miq. and C. tribuloides J.E.Sm. from Nepal can only maintain viability for 2 months in moist storage at room temperature but did not provide any information about viability following desiccation. Tian and Tang (2010) found that fresh seeds of C. fissa with $42.7 \%$ MC had $100 \%$ germination. However, drying seeds to $25.3 \% \mathrm{MC}$ reduced the germination to $17 \%$. Further desiccation to $17.3 \% \mathrm{MC}$ within 72 hours resulted in complete seed death. However the quick drying rate of $C$. fissa seeds is in contrast to that of other Fagaceae species reported by various authors (Xia et al., 2012b; Li et al., 2018). In general, the silica gel and Quercus seeds are mixed and sealed in airtight containers, in a 1:1 to 5:1 ratio by weight (Xia et al., 2012a; Xia et al., 2012b; Li et al., 2018). Seeds dried in silica gel require about 20 days to reach approximately $20 \%$ MC. Recently, Basrudin et al. (2019) reported that the seeds C. buruana Miq. dried in the sunlight after washing reduced viability. Neither the MC measurements nor any desiccation experiments were performed. Apparently, there are no other studies on seed desiccation-sensitivity of Castanopsis.

Seed anatomy plays an important role in controlling water loss of Fagaceae species. The pericarp of Fagaceae seeds was sub-divided into three regions (Kaul, 1985; Xia et al., 2012b): (1) the apex; (2) the scar; and (3) the main pericarp (the remaining area between the apex and the scar region). Using nine Quercus species, Xia et al. (2012b) reported that the water loss occurs (only) through the scar during 
desiccation in many -but not all- species, and the main pericarp being impermeable to water, protects the embryo axis by not allowing the water to leave seeds. In most Quercus seeds, the embryo axis is located on the opposite side of the scar (Bonner and Vozzo, 1987; Finch-Savage and Clay, 1994). Water loss occurs only through the scar and will dry the cotyledons and keep the embryo in a hydrated state for a long-time (Finch-Savage and Blake, 1994). Consequently, this mechanism could protect the seeds under ecological drying due to drought. As such, embryo axis desiccation is avoided until the whole seed cotyledons dries out. Therefore, the embryo position in Quercus aids in survival of seeds during extreme drying conditions. Chen et al. (2000) showed longitudinal section diagrams of the representative seeds/fruits of five genera of Fagaceae, including Castanea mollissima BL., Castanopsis faberi Hance, Cyclobalanopsis pachyloma (Seemen) Schottky, Fagus longipetiolata Seem., Lithocarpus glaber (Thunb.) Nakai and Quercus aliena var. acutiserrata Maximowicz ex Wenzig. The embryo axes of these six species are located at the apex region. As such, it can be expected that they are protected against drying stress during desiccation in the field. However, whether a similar seed anatomy is found across other species of Fagaceae is currently unknown and worth considering for further investigation.

Castanopsis sclerophylla is distributed in southern and eastern subtropical forests of China and is one of the dominant species of evergreen broad-leaved forest (Huang et al., 1999). It is an important 
111

112

113

$\mathrm{X}$-ray computed tomography $(\mathrm{CT})$ is a non-invasive approach that provides 3-D visualization and can quantify biological structures (Foucat et al., 1993; Dell'Aquila, 2007; ISTA, 2020). In Syagrus flexuosa fruits, Stuppy et al. (2003) used high-resolution X-ray computed tomography (HRCT) to clearly distinguish all morphologically relevant parts and organs such as the endocarp with fibrovascular bundles and endosperm with embryo. Kunishima et al. (2020) used a laboratory-based X-ray microscope to study the 3D-structure of pansy (Viola $\times$ wittrockiana $)$ seeds without any pretreatment.

Here, C. sclerophylla endemic to China was selected as a species of the genus Castanopsis and the first objective of this study was to define the germination characteristics and drying pattern of $C$. sclerophylla seeds. In particular, we wanted to investigate seed desiccation tolerance. The second objective was to use CT to scan the whole seed without any treatment, to view the internal structure of seeds and better understand the implications of the morphological structure of seeds for drying. This study provides the description of the anatomical features of $C$. sclerophylla seeds for the first time through CT and anatomical observation, so as to improve the lack of anatomical studies on seeds of Castanopsis species.

\section{Materials and Methods}

Fully matured fruits (acorns; but hereafter referred to as seeds) of C. sclerophylla were collected which was the natural dispersal time during the last week of November 2018 from 11 trees in Thousand Island Lakes (TIL) of Zhejiang province, China (29²2" - 2950" N, $118^{\circ} 34^{\prime \prime}-119^{\circ} 15^{\prime \prime}$ E). Previous field trips showed only a handful number of trees were capable of bearing fruits, therefore only a minimum amount of seeds were collected so that the natural regeneration process is unaltered. The seed collection region of TIL has a temperate climate with a mean annual temperature of $17.6{ }^{\circ} \mathrm{C}$ between 2008 and 
2017, ranging from $5.4{ }^{\circ} \mathrm{C}$ in January to $29{ }^{\circ} \mathrm{C}$ in July. The study site receives an annual rainfall of $1637.46 \mathrm{~mm}$, with peak rainfall occurring between March and June. After collection, the seeds were placed in plastic bags containing holes and brought to the University of Shanghai for Science and Technology, Shanghai, China, within three days. After arrival, any insect infested seeds were removed by visual inspection. Seeds were surface sterilized by soaking in sodium hypochlorite $(\mathrm{NaOCl})$ solution for 30 minutes and washed in sterile water thrice. The seeds were stored at $4{ }^{\circ} \mathrm{C}$ until used in the experiments in plastic bags with holes for gas exchange. Seeds were used in the subsequent experiments within 5 days after collection.

The average weight of 100 seeds was determined by measuring the weight of three replicates of 100 seeds using a standard balance $(0.001 \mathrm{~g})$. MC was determined by drying three replicates of five seeds at $103^{\circ} \mathrm{C}$ for 17 hours in a standard oven drying method, following the methods recommended by the International Seed Testing Association (ISTA, 2020). All MC are expressed on a f. wb.

Given limited seed availability, germination tests were conducted using four replicates of 10 seeds. Many previous studies have used similar, if not, lower number of seeds for germination assessment, due to the recalcitrant nature of Fagaceae species (Pritchard, 1991; Xia et al., 2012a; Xia et al., 2012b; Xia et al., 2015). All the germination tests in this study employed incubating seeds on $1 \%$ agar-water in closed plastic boxes with small holes $(1 \times \mathrm{b} \times \mathrm{w}: 154 \times 95 \times 45 \mathrm{~mm})$ at $15 / 20^{\circ} \mathrm{C}(12$ hours dark / 12 hours light of $60 \mu \mathrm{mol} \mathrm{m} \mathrm{s}^{-2} \mathrm{~s}^{-1}$ light provided by cool tungsten filament bulbs available in the germination chamber). Light was supplied only during the warm phase. Seed germination was defined as radicle emergence by at least $2 \mathrm{~mm}$ and counted weekly until no germination for three consecutive weeks.

Our preliminary trials showed that seeds of C. sclerophylla dried slowly and lost viability at around $25 \%$ MC. Therefore, to properly assess the seed responses to desiccation, a total of 275 seeds were 
155

156

157

grouped into five sets each containing 55 randomly selected seeds and dried separately using freshly regenerated silica gel (2-4 mesh) in a 5:1 ratio by weight. Seeds and silica gel were mixed, sealed in five different airtight polythene boxes $(1 \times \mathrm{b} \times \mathrm{w}: 170 \times 115 \times 45 \mathrm{~mm})$ and placed at room temperature $\left(22 \pm 1^{\circ} \mathrm{C}\right)$. After $3,5,7,14$ and 21 days, one set of seeds (i.e. one box) each were opened and three replicates of five seeds (15 in total) were used to determine the MC of the seeds. The remaining seeds (four replicates of 10) were used for germination as mentioned above and seeds were photographed at different germination stages with a camera (EOS 60D, Canon, Japan). Germination percentage following drying to various $\mathrm{MC}$ were arcsine transformed before analysis and tested for statistical significance $(P<0.05)$ using one-way ANOVA with Duncan test in SPSS, version 21.0.

To determine the morphology, full and cross-sections of the seeds were photographed and the whole seeds were scanned using Industrial Computer Tomography (XTH 225 ICT, Nikon, Japan). The seeds were placed on the CT sample table and moved to the appropriate position. After scanning several times and to improve the clarity of the images, the parameters of CT scan were set as: tube voltage $90 \mathrm{kV}$, tube current $90 \mu \mathrm{A}$, exposure time $500 \mathrm{~ms}$, and the number of pictures taken 2000 times. Using the built-in image processing software (CT Pro 3D, Nikon, Japan), the imported scanned images were reconstructed in three dimensions (3D) to obtain the CT image of the seed, including the front view, the top view and the side view.

\section{Results}

The average 100-seed weight was $134.1 \pm 6.8 \mathrm{~g}$. Freshly collected seeds had a MC of $36.2 \pm 1.9 \%$ and $92.5 \pm 0.4 \%$ germination (Fig. 1). After a 7-day drying period, seed MC decreased to $22.5 \%$ and germination decreased significantly $(\mathrm{P}<0.05)$ to $27.5 \%$. The viability of seeds dried to $20.7 \% \mathrm{MC}$ (seeds 
177 dried for 14 days) was 5\% and further drying to lower MC resulted in complete loss of viability (Fig.

178 1). The sharp decline in germination when seeds $\mathrm{MC}$ was reduced to c. $20 \% \mathrm{f}$. wb. indicates that the 179 seeds are desiccation-sensitive.

The anatomical structure and germination stages of $C$. sclerophylla seed are shown in Figs. 2,3. The pericarp of C. sclerophylla is similar to other Fagaceae seeds, such as Quercus (Xia et al., 2012b) with three distinguishable regions including (1) the apex; (2) the scar; and (3) main pericarp (Figs. 2,3a). The embryo axis is clearly on the opposite side of the apex and sits just below the scar region (Fig. 2). When the seeds germinate, the embryo axis elongates and grows thicker initially before protruding through the scar, then elongating into cotyledonary petiole and radicle (see Figs. 2,3).

\section{Discussion}

Desiccation-sensitive nature of the seeds appears to be a common trait in Fagaceae species across genus.

Compared with other desiccation-sensitive seeds of Fagaceae species, e.g. Q. robur (Finchsavage, 1992), Q. coccifera L., Q. pubescens Willd. and Q. pedunculiflora K. Koch (Ganatsas and Tsakaldimi, 2013), C. sclerophylla seeds had a similar desiccation response, showing that the percent germination of seeds decreased with a decline in MC. The seeds lost their viability completely when the MC of the seeds dropped to less than $20 \%$ in $2-3$ weeks. In particular, the result is consistent with drying rates reported for Quercus seeds, where a relatively slower drying rate has been observed (Xia et al., 2012a). Clearly, the seeds of $C$. sclerophylla required 15-25 days to reach MC level of $20 \%$, which is lethal to seeds when dried in silica gel. However, Tian and Tang (2010) reported that C. fissa and Q. fabri seeds can be dried to approximately $20 \% \mathrm{MC}$ within 72 hours, which is in marked contrast with our results. longer drying period of at least 20 days to reach approximately $20 \%$ when dried in a similar ratio of 
silica gel used in the present study, which is in agreement with Xia et al. (2012b). The contradicting result on the drying rate could be attributed to the differences in the drying method. Whether or not the seeds were dried in a sealed environment and the amount of silica gel used was not provided by Tian and Tang (2010). It must be noted, however, that the silica gel provided a very dry environment that can be only created ex-situ, the natural drying rates might be slower and depend on the environmental relative humidity and temperature.

Xia et al. (2012b) found that water loss during seeds desiccation of Quercus species occurs only through the scar instead of the main pericarp or the apex in some species including $Q$. nuttallii, Q. suber and $Q$. palustris. Xia et al. (2012a) showed that the MC of $Q$. franchetii only reached $23 \%$ after 164 days of drying in silica gel. For this species, a comparatively small scar (ca. $6 \%$ of the pericarp area) might be the mechanistic basis of limited water loss through the pericarp (Xia et al., 2012b). For Q. suber, Eduardo and Belén (2000) studied the morphological and micro-morphological differences of the scar and main pericarp. The main pericarp provides a protective barrier that decreases the rate of water loss, and it mainly consists of a layer of epidermal wax, a thick cuticle covering the epidermal layer and a single layer of palisade cells. However, the scar lacks this protective structure, which consists of a vascular strand presumably facilitating moisture loss. Thus, this feature was reported to have ecological advantage, because the embryos of Quercus seeds cannot reach lower water content during brief exposure to drought conditions in natural environment.

While investigating the route of water loss in C. sclerophylla might be arguably an important area worth exploring, we argue the results of photomicrographs and CT scan suggest otherwise. In all the Fagaceae species, the positioning of embryo on the apex side of the acorns tends to be a common feature (Chen et al. 2000). As such it can be expected that the embryo axes of these species are protected against 
desiccation. However, in C. sclerophylla, the embryo axis is clearly on the opposite side of the apex and sits just below the scar region (Fig. 2). This is in marked contrast with the Quercus embryo axis, which is located on the apex side and far away from the scar (Finch-Savage and Blake, 1994; Xia et al., 2012b). Thus, during desiccation regardless whether water loss only occurs through scar or intact pericarp, the embryo axis of $C$. sclerophylla seeds will be the first part within the seeds to lose water due to its position and continuous drying may kill the seeds. However, our drying results indicate that C. sclerophylla seeds lose viability only when the MC drops to c. $20 \%$ f.wb.

There appears to be a strong difference in the germination pattern between Quercus and C. sclerophylla seeds. Notably, in C. sclerophylla seeds, the radicle protrudes through the scar during germination (see Figs. 2,3), while for Quercus seeds, the radicle protrudes through the apex region (Bonner and Vozzo, 1987; Löf et al., 2019). It is possible to suggest that this difference could be due to the position of the embryo axis between these two genera. However, this requires detailed investigation given that seeds of many Quercus species have epicotyl physiological dormancy (Farmer Jr, 1977; Baskin and Baskin, 2014).

In conclusion, like most Quercus species, seeds of $C$. sclerophylla are desiccation-sensitive and lose viability only when the MC drops to c. $20 \%$ f. wb. Drying rate is similar to those reported for Quercus, indicating high similarity between the genus. For Fagaceae seeds, studies have been restricted to pericarp anatomy and water loss rate on different regions of the seed coat (Eduardo and Belén, 2000; Xia et al., 2012b). Through CT and other observations, we found that position of the embryo axis in $C$. sclerophylla is different from that of Quercus or other genera reported thus far within Fagaceae. Despite this, the seeds showed a similar pattern of desiccation-sensitivity during drying, indicating that the route of water loss may be less important in C. sclerophylla and other species with the same anatomical 
characteristics. However, whether this particular position of seeds embryo axis is common within

Castanopsis genus has not been thoroughly studied and should form a basis for further study.

\section{Declarations}

246 The authors declare no human subjects were involved in this study. Neither any endangered nor any species that are threatened were studied. Funding authorities have no role in experimental design, writing of manuscript or submission. Authors maintain sole responsibility

249 for the paper.

\section{Acknowledgements}

251 We thank Prof. Hugh Pritchard for his discussion on Fagaceae seeds.

252

253

\section{Financial Support}

Financial support by National Science Foundation of China (NSFC) grant number 31750110474 and 32001119 is gratefully acknowledged.

\section{Conflicts of interest/Competing interests}

The authors declare that they have no conflict of interest.

\section{References}

Baskin, C. C., and Baskin, J. M. (2014). "Seeds: ecology, biogeography, and evolution of dormancy and germination," Second/Ed. Elsevier.

Basrudin, Budi, S. W., Achmad, and Sukarno, N. (2019). Characteristics of morphology, anatomy and dormancy breaks of Castanopsis Buruana Miq. Seeds as an endemic plant of Sulawesi, Indonesia. Plant Archives 19, 4101-4108.

Berjak, P., and Pammenter, N. W. (2008). From Avicennia to Zizania: Seed Recalcitrance in 
Bonner, F., and Vozzo, J. (1987). "Seed Biology and Technology of Quercus," U.S. Dept of Agriculture, Forest Service, Southern Forest Experiment Station. of Botany 78, 181-187. 
288

289

290

291

292

293

294

295

296

Ganatsas, P., and Tsakaldimi, M. (2013). A Comparative Study of Desiccation Responses of Seeds of Three Drought-resistant Mediterranean Oaks. Forest Ecology and Management 305, 189-194.

Huang, C. J., Zhang, Y. T., and Bartholomew, B. (1999). "Fagaceae (Vol. 4). Flora of China (FOC) " Science Press, Beijing, China.

ISTA (2020). "The International Seed Testing Association," Bassersdorf, CH-Switzerland.

Kaul, R. (1985). Reproductive Morphology of Quercus (Fagaceae). American Journal of Botany, 72, 1962-1977.

Kunishima, N., Takeda, Y., Hirose, R., Kalasová, D., Šalplachta, J., and Omote, K. (2020). Visualization of internal 3D structure of small live seed on germination by laboratorybased X-ray microscopy with phase contrast computed tomography. Plant Methods 16, 7.

Li, J., Jaganathan, G. K., Zhong, Y., Liu, B., and Yu, M. (2018). Exogenous application of hydrogen peroxide improves germination in slow drying Quercus fabri (Fagaceae) seeds, but does not improve longevity. Seed Science and Technology 46, 377-383.

Löf, M., Castro, J., Engman, M., Leverkus, A. B., Madsen, P., Reque, J. A., Villalobos, A., and Gardiner, E. S. (2019). Tamm Review: Direct seeding to restore oak (Quercus spp.) forests and woodlands. Forest Ecology and Management 448, 474-489.

Pritchard, H. W. (1991). Water Potential and Embryonic Axis Viability in Recalcitrant Seeds of Quercus rubra. Annals of Botany 67, 43-49.

Roberts, E. H. (1973). Predicting the Storage Life of Seeds. Seed Science and Technology 1, 
Schopmeyer, C. S. (tech. coord.) (1974). Seeds of Woody Plants in the United States. Agricultural Handbook No. 450. USDA Forest Service, Washington, DC. Available from: http://data.kew.org/sid/ (May 2020). 

Anatomy. Plos One 7, e47368.

Xia, K., Daws, M. I., Zhou, Z. K., and Pritchard, H. W. (2015). Habitat-linked Temperature Requirements for Fruit Germination in Quercus Species: A Comparative Study of Quercus subgenus Cyclobalanopsis (Asian evergreen oaks) and Quercus subgenus Quercus. South African Journal of Botany 100, 108-113.

Xin, Z., Gaofu, X. U., Dongwei, S., Yongjie, G. U., Hui, G., Xiaohua, L., and Xiaoyong, C. (2007). Maintenance and natural regeneration of Castanopsis sclerophylla populations on islands of Qiandao Lake Region. Acta Ecologica Sinica 27, 424-431. 


\section{$342 \quad$ Figure legends}

343 Figure 1. Effect of silica gel drying on germination of Castanopsis sclerophylla seeds. Different lower-

344 case letters indicate significant difference in germination between seeds dried to various moisture 345 contents $(P<0.05)$. Error bars represent standard deviation of the mean.

346

347

Figure 2. Photomicrographs and CT images of Castanopsis sclerophylla seeds (a) half cotyledon with embryo axis; (b) growth of the embryo axis; (c) top view; (d) front view. SC, seed coat; COT, cotyledons; EMB, embryo axis.

350

351

Figure 3. Photographs of Castanopsis sclerophylla seeds at different stagess of germination (a) whole seed; (b) 0h; (c) 48h; (d) 96h; (e) 144h; (f) 240h. SC, seed coat; SR, scar; AP, apex; R, radicle, COT, cotyledons; S, stem; CP, cotyledonary petiole. 


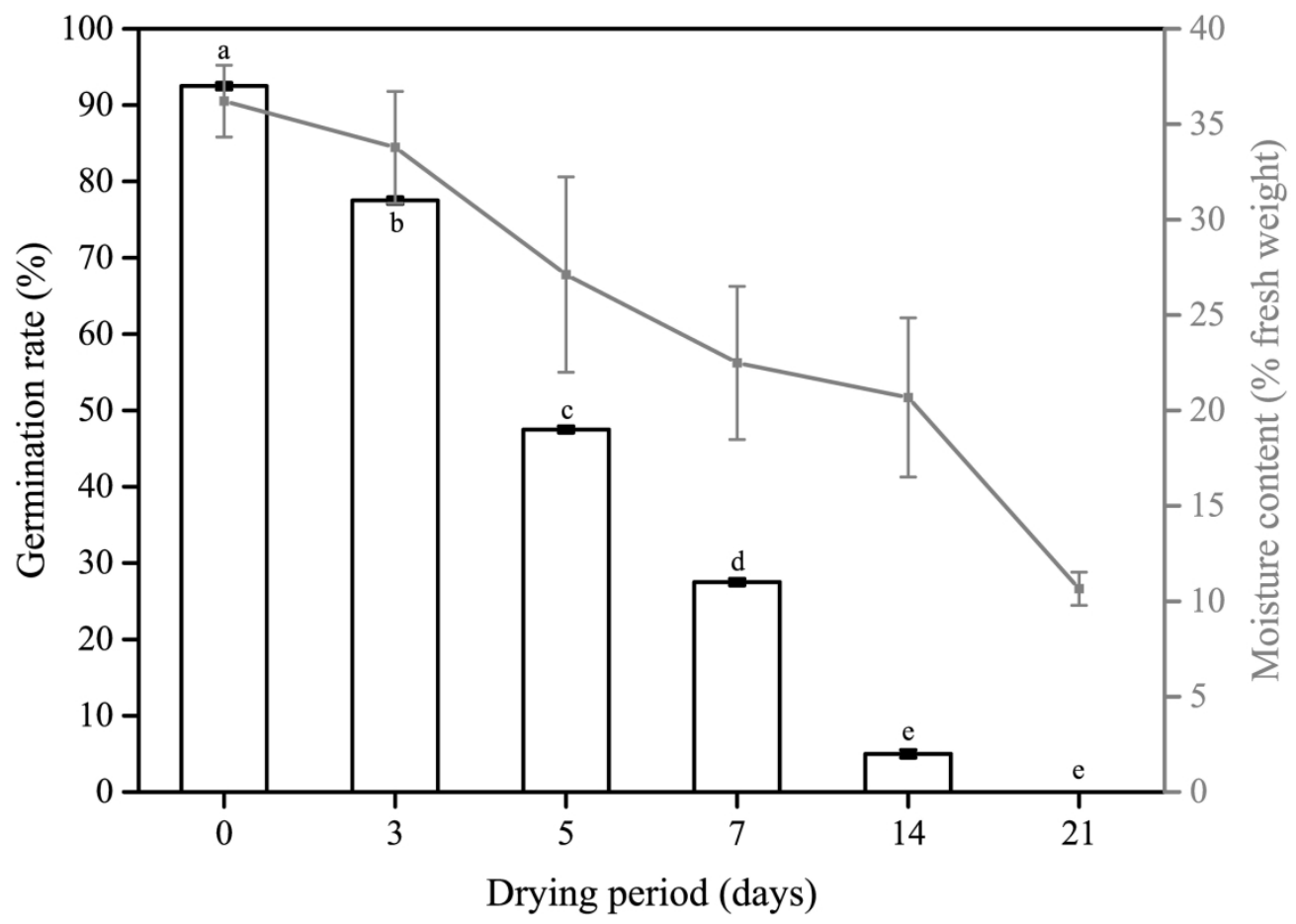

Figure 1. Effect of silica gel drying on germination of Castanopsis sclerophylla seeds. Different lower-case letters indicate significant difference in germination between seeds dried to various moisture contents $(P<$ 0.05). Error bars represent standard deviation of the mean.

$254 \times 180 \mathrm{~mm}(300 \times 300$ DPI) 


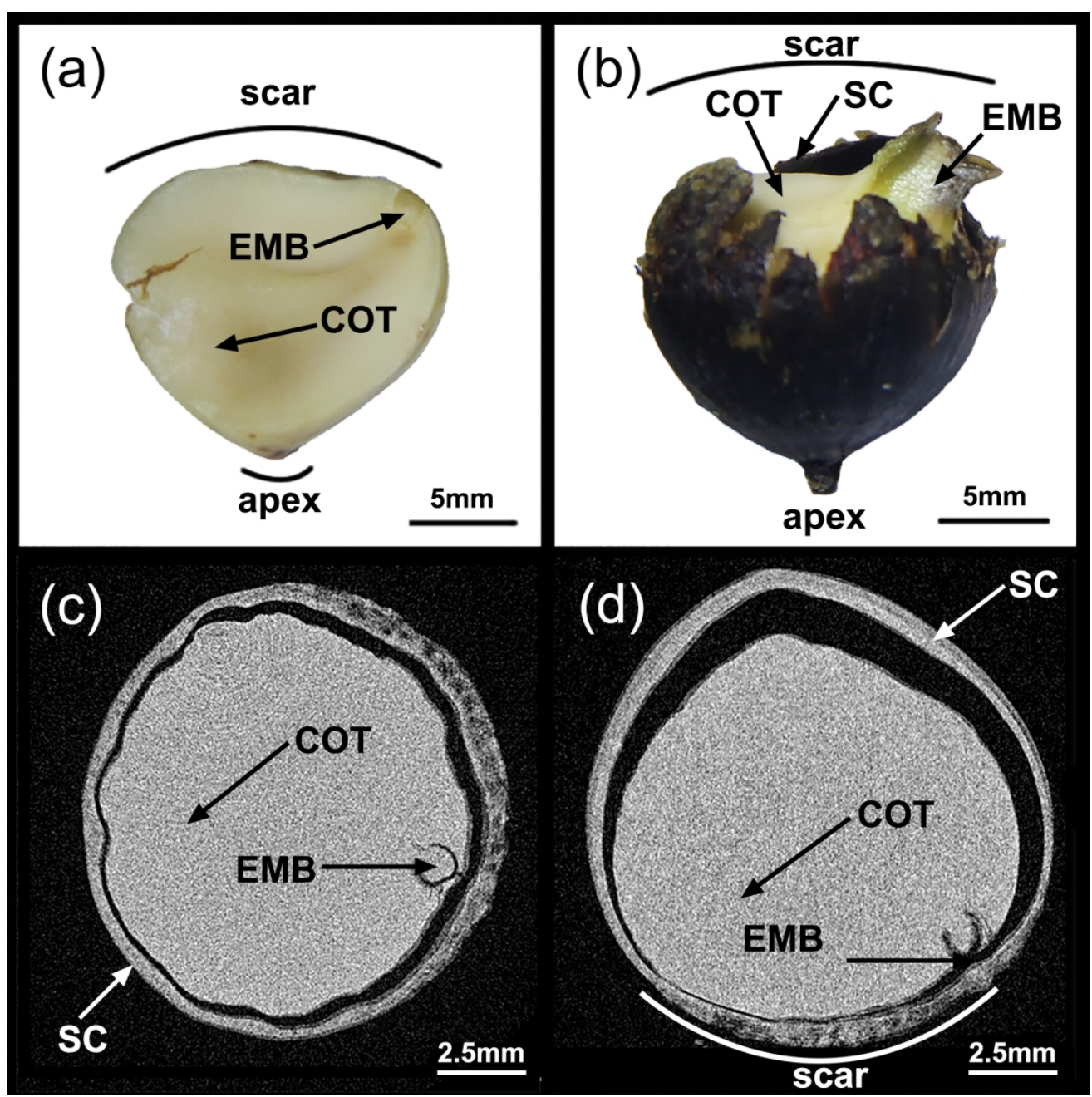

Figure 2. Photomicrographs and CT images of Castanopsis sclerophylla seeds (a) half cotyledon with embryo axis; (b) growth of the embryo axis; (c) top view; (d) front view. SC, seed coat; COT, cotyledons; EMB, embryo axis.

$691 \times 691 \mathrm{~mm}(72 \times 72 \mathrm{DPI})$ 

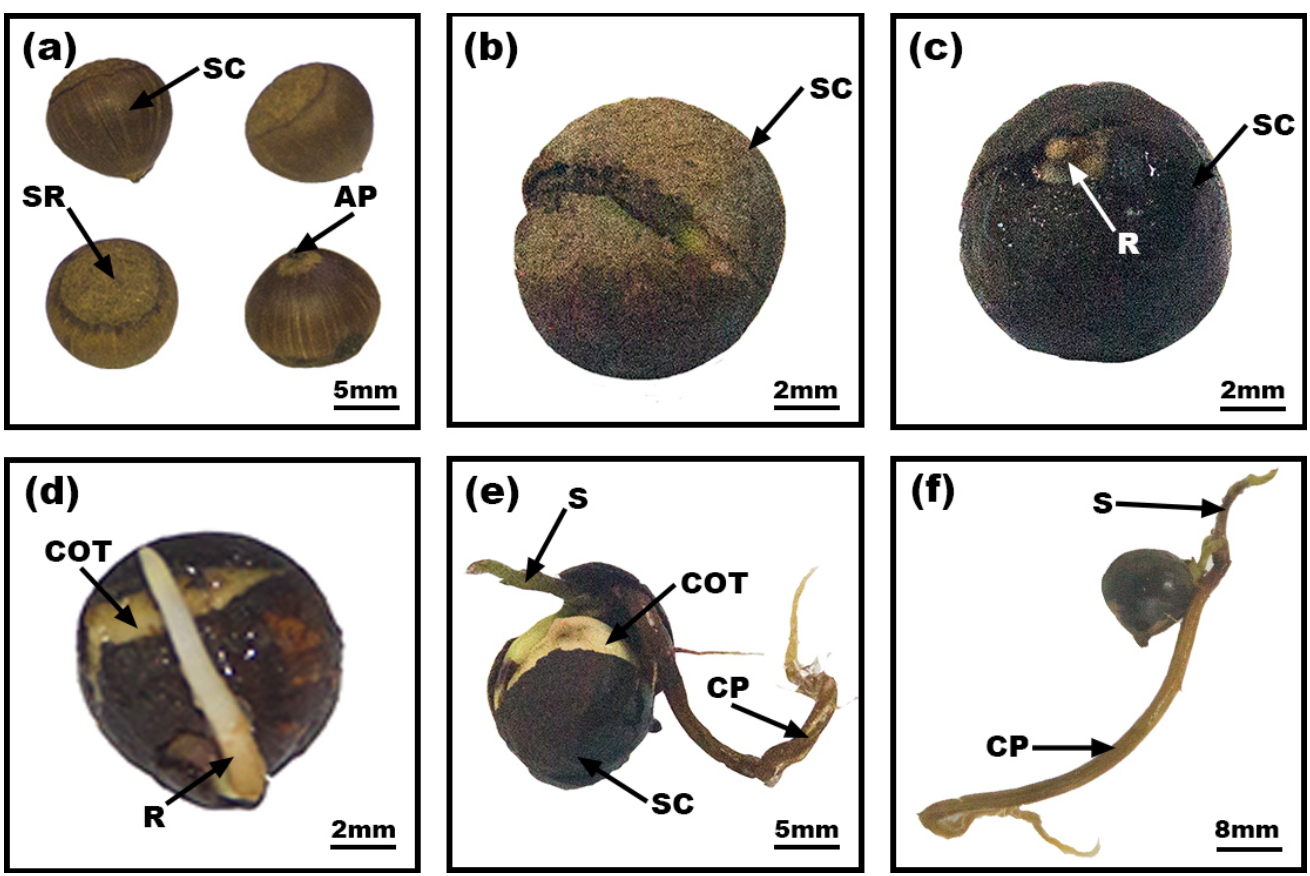

Figure 3. Photographs of Castanopsis sclerophylla seeds at different stagess of germination (a) whole seed;

(b) 0h; (c) 48h; (d) 96h; (e) 144h; (f) 240h. SC, seed coat; SR, scar; AP, apex; R, radicle, COT, cotyledons; S, stem; $\mathrm{CP}$, cotyledonary petiole.

$391 \times 258 \mathrm{~mm}(72 \times 72 \mathrm{DPI})$ 